\title{
Fluorescein Gonioangiography of Newly Formed Vessels in the Anterior Chamber Angle
}

\author{
Ryozo Kimura \\ Department of Ophthalmology, Tohoku University School of \\ Medicine, Sendai 980
}

\begin{abstract}
Kimura, R. Fluorescein Gonioangiography of Newly Formed Vessels in the Anterior Chamber Angle. Tohoku J. exp. Med., 1983, 140 (2), 193-196 Fluorescein gonioangiography was performed in 20 eyes of 20 patients with various ocular disorders such as diabetic retinopathy, retinal vessel occlusion, traumatic angle recession, and granulomatous uveitis. It was clearly demonstrated that newly formed vessels in the anterior chamber angle originated from the ciliary body, and extended predominatingly onto the surface of the angle wall via ciliary body band. gonioangiography; fluorescein angiography; gonioscopy; neovascular glaucoma; anterior chamber angle
\end{abstract}

Neovascular glaucoma is a devastating disorder of the eye resulting from the fibrovascular membrane over the trabecular meshwork which impaires the aqueous outflow. It is well known that retinal ischemia caused by retinal vascular disorders such as diabetic retinopathy and central retinal vessel occlusion are the predisposing factors resulting in neovascularization in the anterior chamber angle. However, because of difficulties to detect fine vessels in the anterior chamber angle by routine gonioscopy, there have been few precise descriptions concerning the pattern of neovascularization in the anterior chamber angle. The author (1980) developed a new effective method of fluorescein gonioangiography. Among several contributions afforded using the method (Kimura 1982; Kimura et al. 1982; Asano and Kimura 1983;), the method made angle vessels accessible which were hardly detectable in routine gonioscopy (Kimura 1980). Thus, it seems worthy to evaluate the pattern of newly formed vessels in the anterior chamber angle by means of the fluorescein gonioangiography.

\section{Subjects And Methods}

A method of fluorescein gonioangiography (Kimura 1980) was applied in this study. 20 patients with various ocular disorders volunteered from our clinic. The subjects (20 eyes) consisted of 10 eyes of 10 patients with diabetic retinopathy, 3 eyes of 3 patients after retinal vessel occlusion, 2 eyes of 2 patients with traumatic angle recession, and 5 eyes of 5 patients with granulomatous uveitis. Prior to performing fluorescein gonioangiography, a complete eye examination was performed. Of 20 eyes studied, 2 eyes with diabetic retinopathy and an eye with retinal vessel occlusion showed increased intraocular pressure. All diabetics studied had proliferative retinopathy of various stages. Of 3 eyes with retinal

Received for publication, October 16, 1982. 
vessel occlusion, an eye had a history of central retinal artery occlusion, and remaining 2 eyes had a history of central retinal vein occlusion. Both of the 2 eyes with traumatic angle recession had sustained a contusive eye injury several years before. All eyes with granulomatous uveitis had a protracted course of uveitis: They consisted of 3 eyes of ocular sarcoidosis, and 2 eyes of Vogt-Koyanagi-Harada syndrome.
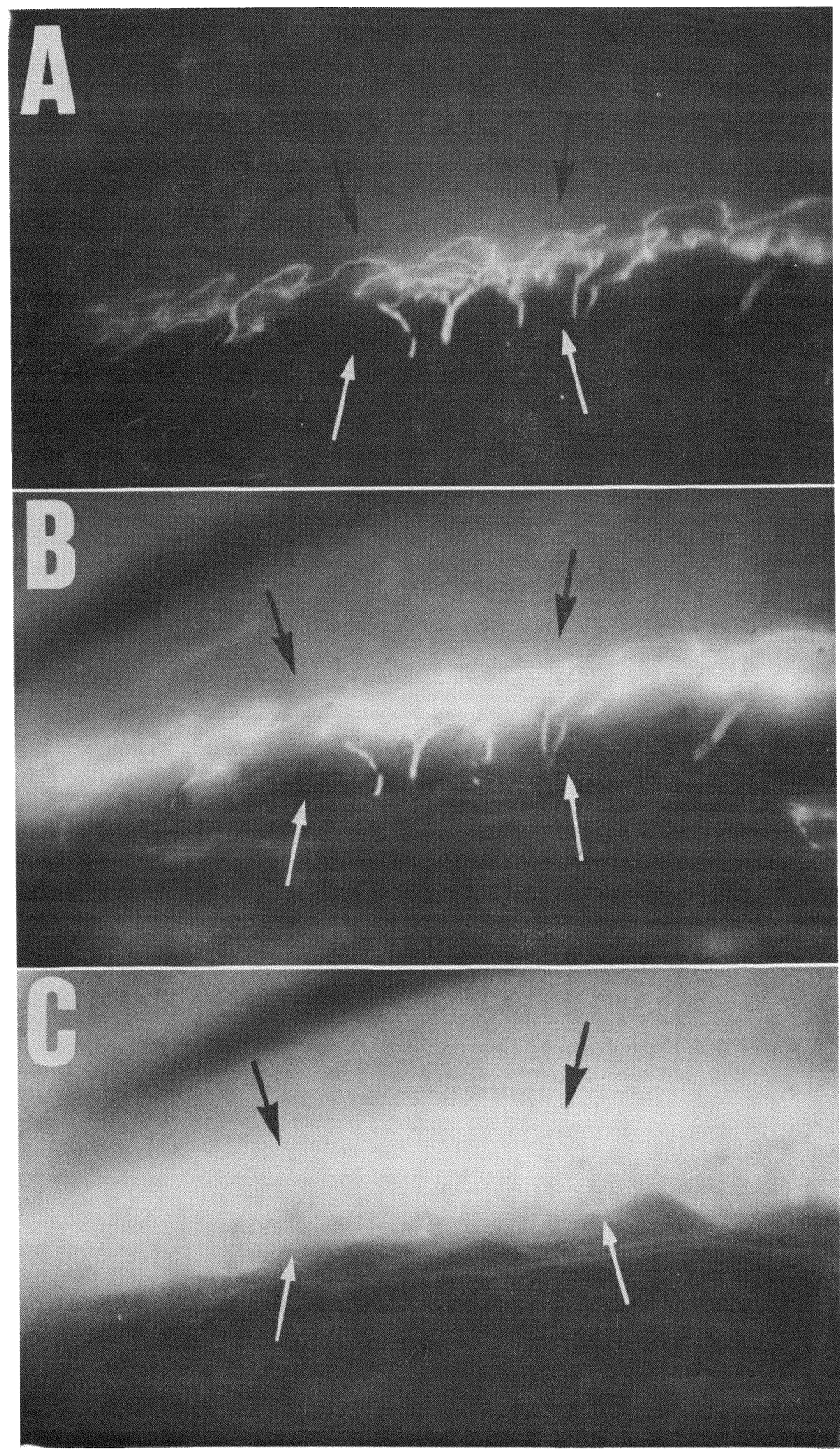

Fig. 1. Fluorescein gonioangiograms in a patient with proliferative diabetic retinopathy, (A) in the early phase, and (B and C) in the late phase of the angiography. White arrows indicate the iris root, and black arrows indicate the Schwalbe's line. Note even in such an advanced case, newly formed angle vessels which show leakage in the late phase of the angiography are almost restricted only on the surface of the angle wall. 


\section{Results}

In all eyes studied, newly formed angle vessels which were hardly detectable in routine gonioscopy were clearly demonstrated by means of the fluorescein gonioangiography. Newly formed angle vessels fluoresced in the early phase of the angiography, and leaked fluorescein in the late phase of the angiography (Figs. 1, 2). It was also clear that newly formed angle vessels originated from the ciliary body. Table 1 summarizes the site of neovascularization in the anterior chamber angle of 20 eyes studied; all eyes showed newly formed vessels on the surface of the angle wall, while only three eyes $(15 \%)$ showed newly formed vessels on the surface of the peripheral iris.

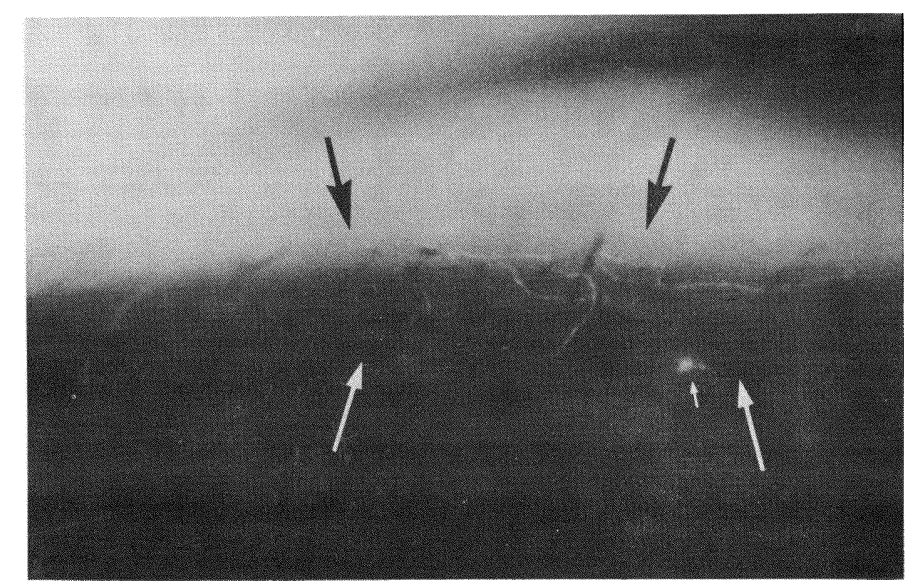

Fig. 2. Fluorescein gonioangiogram of a protracted case of ocular sarcoidosis in the early phase of the angiography. White arrows indicate the iris root, and black arrows indicate the Schwalbe's line. A small white arrow indicates the granulomatous lesion which shows hyperfluorescence. Note fine newly formed angle vessels on the angle wall which are hardly detectable in routine gonioscopy.

TABLE 1. Site of neovascularization among 20 eyes studied

\begin{tabular}{lccc}
\hline \multicolumn{1}{c}{ Diagnosis } & $\begin{array}{c}\text { Number } \\
\text { of } \\
\text { eyes }\end{array}$ & $\begin{array}{c}\text { Site of neovascularization } \\
\text { (eyes) }\end{array}$ & $\begin{array}{c}\text { Peripheral iris } \\
\text { (eyes) }\end{array}$ \\
\hline Diabetic retinopathy & 10 & 10 & 2 \\
Retinal vessel occlusion & 3 & 3 & 1 \\
Traumatic angle recession & 2 & 2 & 0 \\
Granulomatous uveitis & 5 & 5 & 0 \\
\hline
\end{tabular}

\section{Discussion}

As described by Chandler and Grant (1979), it is widely accepted that new vessels in the anterior chamber angle grow from the circumferential artery of the ciliary body onto the surface of the iris and on the surface of the wall of the angle. In the present study, however, it was clearly demonstrated that newly formed angle 
vessels which originated from the ciliary body, extended predominatingly onto the surface of the angle wall. This discrepancy in distribution of newly formed angle vessels may be attributed to the method of examination adopted: Chandler and Grant (1979) as well as all other investigators examined by routine gonioscopy, while the author examined by the fluorescein gonioangiography which made even fine vessels accessible. Thus, importance to perform fluorescein gonioangiography in patients with retinal vascular disorders such as diabetic retinopathy and central retinal vessel occlusion was stressed so as to select effective procedures as early as possible.

\section{References}

1) Asano, Y. \& Kimura, R. (1983) Glaucoma associated with Sturge-Weber syndrome A case report. Glaucoma (Chicago), 5, in press.

2) Chandler, P.A. \& Grant, W.M. (1979) Glaucoma. 2nd ed., Lea \& Febiger, Philadelphia, p. 45 .

3) Kimura, R. (1980) Fluorescein goniophotography. Glaucoma (Chicago), 2, 359-367.

4) Kimura, R. (1982) Hyperfluorescent dots in the ciliary body band in patients with granulomatous uveitis. Brit. J. Ophthalmol., 66, 322-325.

5) Kimura, R., Takaku, Y. \& Suzuki, K. (1982) Aneurysms in the ciliary body band confirmed by fluorescein gonioangiography. Brit. J. Ophthalmol., 66, 725-727. 